\title{
Postural Orthostatic Tachycardia Syndrome
}

National Cancer Institute

\section{Source}

National Cancer Institute. Postural Orthostatic Tachycardia Syndrome. NCI Thesaurus.

Code C85020.

A syndrome characterized by orthostatic intolerance resulting in tachycardia, often accompanied by a decrease in the blood pressure. It occurs when individuals change from the supine position to an upright position. Other signs and symptoms include lightheadedness, fainting, fatigue and muscle weakness. 